\section{Systemic lupus erythematosus and primary fibromyalgia can be distinguished by testing for cell-bound complement activation products}

\author{
Daniel J Wallace, ${ }^{1,2}$ Stuart L Silverman, ${ }^{1}$ John Conklin, ${ }^{3}$ Derren Barken, ${ }^{3}$ \\ Thierry Dervieux ${ }^{3}$
}

To cite: Wallace DJ, Silverman SL, Conklin J, et al. Systemic lupus erythematosus and primary fibromyalgia can be distinguished by testing for cell-bound complement activation products. Lupus Science \& Medicine 2016;3: e000127. doi:10.1136/lupus2015-000127

- Additional material is available. To view please visit the journal (http://dx.doi.org/ 10.1136/lupus-2015-000127)

Received 22 September 2015 Revised 19 November 2015 Accepted 8 December 2015

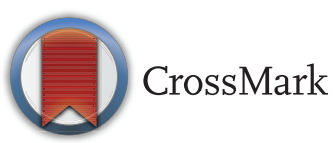

${ }^{1}$ Cedars Sinai Medical Center, Los Angeles, California, USA ${ }^{2}$ Wallace Rheumatology Study Center, Los Angeles, California, USA

${ }^{3}$ Exagen Diagnostics, Vista, California, USA

Correspondence to Dr Thierry Dervieux; tdervieux@exagen.com Dr Daniel Wallace; danielwallac@gmail.com

\section{ABSTRACT}

Objective: We sought to establish the performance of cell-bound complement activation products (CB-CAPs) as a diagnostic tool to distinguish primary fibromyalgia (FM) from systemic lupus erythematosus (SLE).

Methods: A total of 75 SLE and 75 primary FM adult subjects who fulfilled appropriate classification criteria were enrolled prospectively. CB-CAPs (erythrocyte-C4d (EC4d) and B-lymphocyte-C4d (BC4d)) were determined by flow cytometry. Antinuclear antibodies (ANAs) were determined using indirect immunofluorescence while other autoantibodies were determined by solid-phase assays. The CB-CAPs in a multi-analyte assay with algorithm (MAAA) relied on two consecutive tiers of analysis that was reported as an overall positive or negative assessment. Test performance was assessed using sensitivity, specificity, positive and negative likelihood ratio (LR).

Results: ANAs yielded $80 \%$ positives for SLE and $33 \%$ positives for FM. High CB-CAP expression (EC4d $>14$ units or BC4d $>60$ units) was $43 \%$ sensitive and $96 \%$ specific for SLE. The CB-CAPs in MAAA assessment was evaluable in 138 of the 150 subjects enrolled (92\%) and yielded $60 \%$ sensitivity (CI 95\% $48 \%$ to $72 \%$ ) for SLE with no FM patient testing positive $(100 \%$ specificity). A positive test result was associated with a strong positive LR for SLE (>24, Cl $95 \%$; 6 to 102), while a negative test result was associated with a moderate negative LR $(0.40 ; \mathrm{Cl} 95 \%$ 0.30 to 0.54 ).

Conclusion: Our data indicate that CB-CAPs in MAAA can distinguish FM from SLE.

\section{INTRODUCTION}

Systemic lupus erythematosus (SLE) remains the prototypical autoimmune systemic disease in which hyperactivity of the immune system and production of autoantibodies lead to a variety of symptoms including chronic pain, arthralgia, fatigue, morning stiffness and, most importantly, damage in key organs including the kidney and central

\section{KEY MESSAGES}

CB-CAPS in MAAA can distinguish SLE from primary fibromyalgia with $100 \%$ specificity. CB-CAPS are sensitive and specific for SLE.

nervous system. ${ }^{1}$ Because SLE is a serious chronic condition associated with significant mortality and burden to the healthcare system, early diagnosis and initiation of appropriate therapy (eg, immunosuppressants, antimalarials and corticosteroids) is important. ${ }^{2}$ However, many patients with SLE present with symptoms that are non-specific, do not fulfil formal classification criteria of the disease (eg, American College of Rheumatology (ACR) criteria) ${ }^{3}$ and thus may remain undiagnosed for a prolonged time.

This challenge in correctly identifying and diagnosing SLE is further complicated by the low prevalence of the disease $(\sim 1 / 1000$ in the USA) in comparison with other more prevalent rheumatic disorders whose symptoms mimic those of $\mathrm{SLE}^{45}$ such as rheumatoid arthritis, and primary fibromyalgia (FM) ${ }^{6}$ In particular, SLE can sometimes be difficult to identify and differentiate from $\mathrm{FM}$, because the prevalence of FM is at least 10 -fold greater than that of SLE, and most symptomatic patients (eg, with chronic widespread pain) are initially evaluated by primary care physicians who lack expertise in diagnosing the disease. ${ }^{7}$ Moreover, FM is a non-inflammatory pain syndrome, ${ }^{89}$ whereas SLE is a systemic inflammatory illness and thus there a significant treatment and prognostic differences between those two conditions.

Traditionally, clinicians distinguish SLE from other illnesses by a combination of clinical history, demographic, age at disease onset together with examination and the 
determination of laboratory tests that include antinuclear antibody (ANA) among other SLE-specific autoantibodies. ANA is a sensitive test for SLE, and more than 95\% of SLE are ANA positive during the course of their disease. ${ }^{10}$ However, about $14 \%$ of the general population is also ANA positive, ${ }^{11}$ and $15 \%-25 \%$ of patients with FM have been reported to be positive for ANA. ${ }^{12}{ }^{13}$ It follows that the risk of misdiagnosing SLE could be significant, as the vast majority of patients presenting with symptoms mimicking SLE will turn out to have FM and not SLE, even if the ANA test is positive. Other diagnostic tests such as anti-double-stranded DNA (anti-dsDNA) and anti-Smith (anti-Sm) can be helpful in diagnosing SLE, but their utility is limited by their poor sensitivity.

The value of cell-bound complement activation products (CB-CAPs, including C4d deposited on erythrocytes (EC4d) and B-lymphocytes (BC4d) in the differential diagnosis of SLE compared with other autoimmune rheumatic diseases) has been established previously. ${ }^{14-17}$ We recently combined these CB-CAP biomarkers with standard rheumatic disease autoantibodies into a multi-analyte assay with algorithm (MAAA). The CB-CAPs in MAAA have demonstrated improved sensitivity compared with anti-DNA antibodies and low serum levels of C3 and C4 for the diagnosis of SLE. ${ }^{18}$ In the present study, our objective was to analyse the utility of CB-CAPs in MAAA in differentiating SLE from FM.

\section{METHODS}

Two rheumatology practices in the Los Angeles (California, USA) area participated in the enrolment of consecutive adult patients with SLE (who fulfilled the 1982 ACR classification criteria (revised in 1987) for $\mathrm{SLE}^{10}$ or the 2012 Systemic Lupus International Clinic criteria $^{19}$ ), and consecutive adult patients with FM who fulfilled the 1990 ACR criteria). ${ }^{20}$ Patients with SLE having diagnosed FM were excluded. The study was approved by central institutional review boards and all subjects provided informed consent. Patients were compensated for their participation. At time of the study visit, patient-centred outcomes (PCOs) consisting of the Lupus Impact Tracker (LIT) ${ }^{21}$ and Revised Fibromyalgia Impact Questionnaire (FIQ $)^{22}$ were collected for all subjects. Among patients with SLE, disease activity was determined using the SELENA-SLEDAI. ${ }^{23}$ In order to minimise potential bias during patient data collection, the LIT was renamed as Impact tracker, while the FIQ was renamed as Impact questionnaire on case report forms.

Blood was collected in EDTA-containing tubes and serum separator tubes and specimens were shipped overnight to our reference laboratory accredited by the College of American Pathologists and located in Vista, California, USA. ANA was determined by indirect immunofluorescence (IIF) using a Hep-2 substrate and digital scanning on the NOVA view instrument (Inova Diagnostics, San Diego, California, USA). ${ }^{24}$ The cut-off for ANA positivity was set at a titre greater than 1:40. ANA was also determined using ELISA (Quantalyte, Inova Diagnostics). Other autoantibodies including anti-dsDNA, anti-Sm, anti-mutated citrullinated vimentin (anti-MCV), anti-SS-B, anti-Centromere (CENP), anti-Jo-1 and anti-Scl70 were determined as described. ${ }^{18}$ EC4d and BC4d levels were determined by quantitative flow cytometry (and expressed as net mean fluorescence intensity (MFI)) using a laboratory-developed test validated in our clinical laboratory. ${ }^{15}$ All clinical laboratory testing personnel were blinded to patient diagnosis throughout the study duration and all study specimen were processed on the day of receipt with the daily clinical laboratory workload. Serum complement C3 and C4 levels were determined by standard immunoturbidimetry assay (The Binding Site, San Diego, California, USA) and the cut-offs provided by the manufacturer were used for reduced complement level.

The CB-CAPs in MAAA was calculated as per the standard operating procedures in our clinical laboratory and currently approved by the clinical evaluation programme from New York Department of Health. The performance characteristics of the CB-CAPs in MAAA have been described in detail elsewhere. ${ }^{18}$ Briefly, our diagnostic immunology method relies on two consecutive tiers of analysis. In the first tier, positivity for any of anti-dsDNA (>301 units confirmed using the Crithidia Luciliae IIF assay), anti-Sm ( $>10$ Units), elevated EC4d $(>75$ net MFI) or BC4d ( $>200$ net MFI) are associated with a positive test result. All subjects negative in tier 1 are further evaluated in a second tier of analysis (tier 2) that consists of an index score (calculated as an output of multivariate logistic regression) combining an ANA component (using ANA cut-offs at 20 and 60 units, as determined by ELISA), a CB-CAP component (sum of $\log$ normalised EC4d and BC4d net MFI) and an antibody specificity component (corresponding to positivity to either anti-MCV, anti-SS-B, anti-CENP, anti-Scl-70 or anti-Jo-1). Positivity for the tier 2 index score is associated with a positive test result. For each patient specimen, an overall CB-CAPs in MAAA test result that consists of a positive assessment (tier 1 positive or tier 2 index score positive), a negative assessment (tier 2 index score negative), an indeterminate assessment (if the tier2 index score ranges from -0.1 to 0.1 ) or an equivocal assessment (if certain criteria around cut-offs in the index score are not met) is produced. Altogether, the overall sensitivity for SLE and specificity in distinguishing SLE from other diseases has been established at $80 \%$ and $86 \%$, respectively. ${ }^{18}$

Statistical analysis consisted of the determination of test sensitivity and specificity. Positive likelihood ratio (LR) for SLE was expressed as the probability of a SLE subject to test positive for the CB-CAPs in MAAA divided by the probability of a FM subject to also test positive (sensitivity/1-specificity). Conversely, the negative LR was expressed as the probability of a SLE subject to test negative divided by the probability of a FM subject to 
also test negative (1-sensitivity/specificity). Post-test probability for SLE or FM was calculated at various pretest probabilities using Bayesian principles. ${ }^{25}$ LR greater than 10 (or conversely lower than 0.1 ) were interpreted as strong, LR ranging from 5 to 10 (or conversely ranging from 0.2 to 0.1 ) were interpreted as moderate and LR ranging from 2 to 5 (or conversely ranging from 0.2 to 0.5 ) were interpreted as small. LR ranging from 0.5 to 2.0 were considered as having limited to no diagnostic value. ${ }^{26}{ }^{27}$ For some markers, a specificity of $100 \%$ was observed, which makes inference problematic ${ }^{28} 29$ and leads to an infinite positive LR. In those cases, an adjusted specificity of $>97.5 \%$ was estimated, based on the adjusted Wald interval estimation method of Agresti and Coull ${ }^{30} 31$ Tests for statistically significant differences between groups were calculated using Student's t test or Fisher's exact test, as appropriate. Correlations were evaluated using Pearson's product-moment coefficient. CIs for proportions were calculated using a normal approximation interval.

\section{RESULTS}

From July 2014 to January 2015 a total of 150 consecutive subjects including 75 SLE ( $88 \%$ women) and 75 FM (96\% women) were enrolled in the study. All SLE subjects enrolled met both the 1982 ACR and 2012 Systemic Lupus Collaborating Clinic (SLICC) criteria. Patients' demographics are presented in table 1. Online supplementary tables S1 and S2 provide the characteristics of the ACR and SLICC criteria for the SLE subjects. There was a higher percentage of African Americans with SLE than with FM as might be expected from this urban population. Patients with SLE were significantly younger than patients with FM. Patients with FM presented with worse functional status than SLE as determined by PCO (LIT and FIQ) collected in this study. Among SLE, the mean SELENA-SLEDAI was 6.3 \pm 3.0 (SD) (online supplementary table S3).

ANA as determined by IIF ( $>1: 40$ titre) yielded $80 \%$ positives for SLE and 33\% positives for FM (positive

\begin{tabular}{|c|c|c|c|}
\hline & SLE & $\mathbf{F M}$ & p Value \\
\hline Age (years) & $48 \pm 14$ & $53 \pm 14$ & 0.022 \\
\hline Gender (\% female) & $88 \%$ & $96 \%$ & 0.072 \\
\hline \multicolumn{4}{|l|}{ Ethnicity } \\
\hline Caucasians & $57 \%$ & $76 \%$ & \\
\hline African Americans & $23 \%$ & $7 \%$ & 0.019 \\
\hline Hispanic & $11 \%$ & $13 \%$ & \\
\hline Asian/Pacific Islander & $9 \%$ & $4 \%$ & \\
\hline Duration of disease (months) & $13 \pm 10$ & $12 \pm 9$ & 0.504 \\
\hline Lupus Impact Tracker & $41 \pm 22$ & $63 \pm 19$ & $<0.001$ \\
\hline FM Impact Questionnaire & $39 \pm 22$ & $64 \pm 20$ & $<0.001$ \\
\hline
\end{tabular}

$\mathrm{LR}=2.4$, CI $95 \% 1.7$ to 3.4 ; negative $\mathrm{LR}=0.30$, CI $95 \%$ 0.19 to 0.48 ). While the percentage of ANA-positive SLE was relatively low, all patients with SLE enrolled fulfilled the immunological ANA criteria from SLICC and ACR classification criteria and thus had a past history of ANA positivity. ANA (as determined by solid-phase ELISA) was positive ( $\geq 20$ units $/ \mathrm{mL}$ ) in $83 \%$ of SLE and $28 \%$ of FM at the time of the study visit (positive $\mathrm{LR}=3.0$, CI $95 \% 2.0$ to 4.3 ; negative $\mathrm{LR}=0.24$, CI $95 \% 0.14$ to 0.40 ). Altogether, a total of 55 SLE $(73 \%)$ were positive for ANA by both solid-phase assay and IIF, while 14 FM were positive for ANA by both solid phase and IIF (19\%). (positive $\mathrm{LR}=4.0$, CI 95\% 2.4 to 6.0 ; negative $\mathrm{LR}=0.33$, CI $95 \% 0.22$ to 0.48$)$. A total of 67 of the SLE (89\%) were positive for at least one of the two methodologies, while 32 of the FM (43\%) were positive for ANA by at least one of the methodologies (positive $\mathrm{LR}=2.0$, CI $95 \%$ 1.6 to 3.0; negative $\mathrm{LR}=0.19$, CI $95 \% 0.09$ to 0.37 ).

Patients with SLE presented with significantly higher EC4d levels (13.6 \pm 2.4 net MFI, $n=75$, average \pm SEM) than those with FM $(5.5 \pm 0.4$ net MFI, $\mathrm{n}=75$, average \pm SEM $)(p<0.01)$. Sensitivity and specificity for EC4d $>14$ net MFI was $24 \%$ and $96 \%$, respectively (positive $\mathrm{LR}=6.0$, CI $95 \% 1.8$ to 19.5 ; negative $\mathrm{LR}=0.79$, CI $95 \%$ 0.69 to 0.91). Similarly, BC4d levels were higher in SLE than in FM $(96.9 \pm 32.0 \quad(\mathrm{n}=72) \quad$ vs $14.2 \pm 0.7$ net MFI $(\mathrm{n}=75), \mathrm{p}<0.001)$. For three patients with SLE, BC4d was not measurable $(<200$ cell events (CD19+) collected on the flow cytometer) because B-cell depleting therapy (rituximab) was administered to these subjects. Sensitivity and specificity for BC4d $>60$ net MFI was $33 \%$ and $100 \%$, respectively (positive LR $>13$, CI $95 \% 3.1$ to 57 ; negative $\mathrm{LR}=0.67$, CI $95 \% 0.57$ to 0.78 ).

As presented in table 2, low C3/C4 $(\mathrm{C} 3<81 \mathrm{mg} / \mathrm{dL}$ and/or $\mathrm{C} 4<13 \mathrm{mg} / \mathrm{dL}$ ) was observed in $23 \%$ of patients with SLE compared with $1 \%$ of patients with FM, while high CB-CAPs (EC4d $>14$ net MFI or BC4d $>60$ net MFI) were detected in $43 \%$ of patients with SLE compared with $4 \%$ of patients with FM. Positivity for anti-dsDNA as confirmed by the Crithidia Luciliae indirect IIF was low in this SLE cohort (17\%; all ANA positive) but was $100 \%$ specific (one patient with FM presented with reactivity to dsDNA by ELISA which was not confirmed by IIF). All other autoantibodies measured (anti-MCV, anti-SS-B, anti-Jo-1, anti-Scl-70, anti-Sm, anti-CENP) were negative in the group of patients with FM with the exception of one subject positive for anti-CENP. Conversely, among SLE, six of them were positive for anti-Sm $(8 \%)$, six were positive for anti-SS-B $(8 \%)$, three were positive for anti-CENP (4\%) and two were positive for anti-MCV (>70 units; 3\%). EC4d levels were associated with the SELENA-SLEDAI $(\mathrm{r}=0.321 ; \mathrm{p}=0.005)$. BC4d levels did not correlate with disease activity $(\mathrm{r}=0.099 ; \mathrm{p}=0.410)$.

The overall assessment for the CB-CAPs in MAAA was evaluable in 138 of the 150 subjects enrolled (92\%). For 12 subjects the overall assessment was indeterminate (five subjects: four SLE and one FM), equivocal (five 


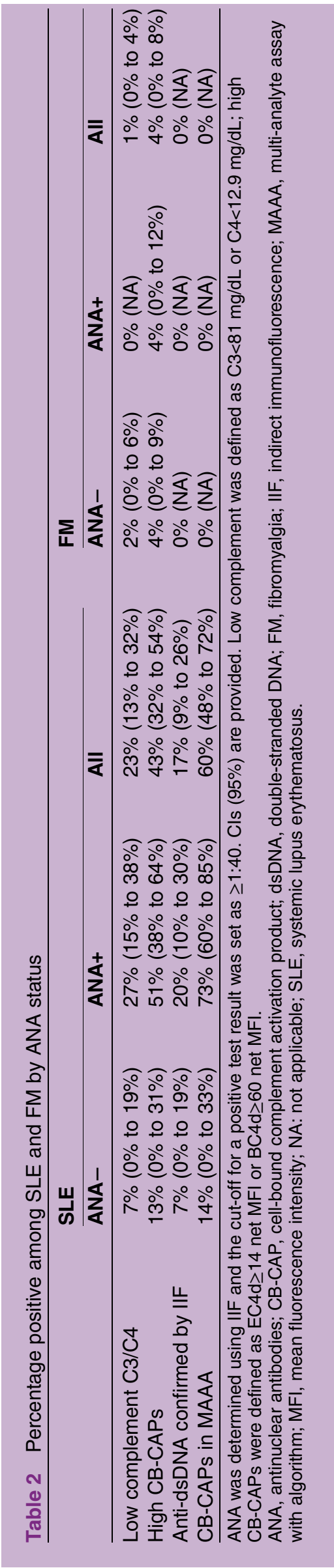

subjects: four SLE and one FM) or non-evaluable because of low B-cell events (two SLE) as described above. As presented in table 2, the CB-CAPs in MAAA yielded a positive test results for $60 \%$ of patients with SLE, while all FM subjects tested negative. Because the proportion of ANA-negative subjects (as determined by IIF using a cut-off for positivity $\geq 1: 40$ ) was significant in this population of SLE subjects (20\%), we also stratified the performances by ANA status. The analysis revealed that the CB-CAPs in MAAA yielded $73 \%$ positive among ANA-positive SLE versus 14\% among ANA-negative SLE subjects. Similarly, higher sensitivity was observed for low complement and high CB-CAPs in ANA-positive compared with ANA-negative SLE subjects.

Table 3 highlights positive and negative LRs for SLE for the CB-CAPs in MAAA and other diagnostic measures. A positive CB-CAPs in MAAA test result was associated with a strong positive LR (>24.0). As such, a positive test result was observed greater than 24 times more frequently in subjects with SLE than in those with FM. Overall, the CB-CAPs in MAAA yielded greater positive LR than traditional low complement $(\mathrm{LR}=17)$, high CB-CAPS (LR=11) and anti-dsDNA (LR >6.9). When the analysis was stratified by ANA status, low complement and high CB-CAPs resulted in lower positive LR among ANA-negative subjects ( $L R=3.3$, in both cases) when compared with ANA-positive subjects (LR >10.7). However, the CB-CAPs in MAAA yielded positive LR greater than 5.7 for negative ANA status and greater than 29 for positive ANA status.

Conversely, a negative CB-CAPs in MAAA test result was associated with a moderate negative LR (0.40). Therefore, a negative test results was observed 2.5 times more frequently in subjects with FM than in those with SLE. However, only the CBCAPs in MAAA yielded negative LR lower than 0.5 (moderate LR) when compared with other diagnostic measures. Negative LR stratified by ANA status indicated a good diagnostic value of CB-CAPs in MAAA among ANA-positive patients (negative $\mathrm{LR}=0.27$ ) in comparison with low complement and anti-dsDNA (LR $>0.7)$. However, none of the diagnostic test presented with negative LR lower than 0.5 among ANA-negative subjects.

Finally, we calculated the post-test probability of SLE (vs FM) at various pretest probabilities by ANA status. As indicated in figure 1 , when the pretest probability of SLE was set at $20 \%$, a positive CB-CAP in MAAA increased the probability of SLE to almost $90 \%$ in ANA-positive patients and to about $60 \%$ in ANA-negative patients. Overall and consistent with the high positive LR, substantial increase in the post-test probability of SLE $(>35 \%)$ was observed irrespective of the ANA status when the CB-CAPs in MAAA test result was positive.

\section{DISCUSSION}

This study builds on our initial observation that CB-CAPs in MAAA add significant value to accurate 


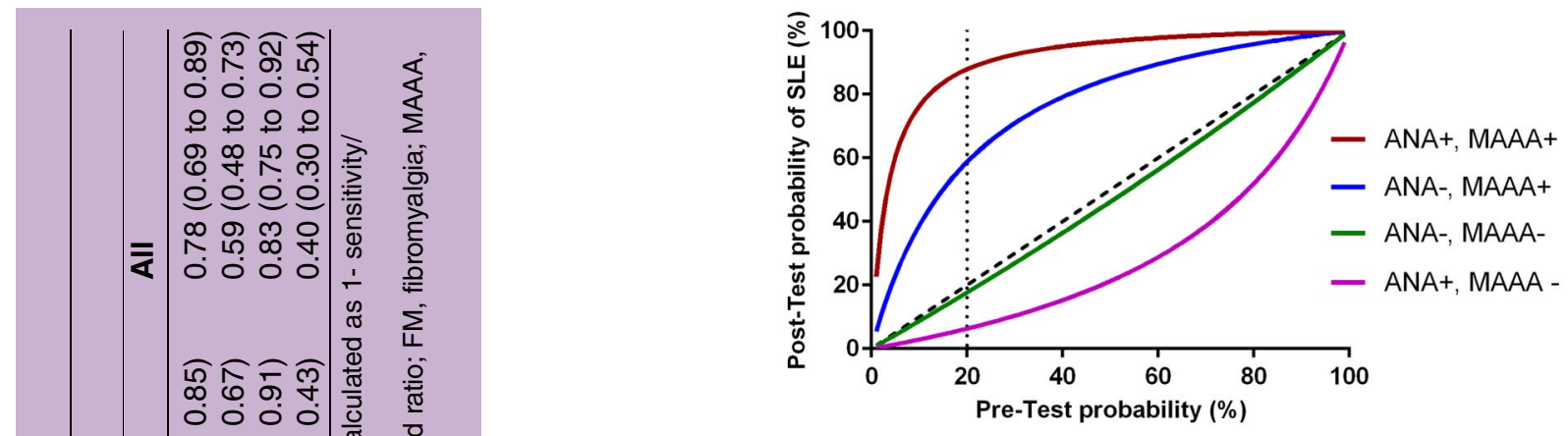

Figure 1 Post-test probabilities of systemic lupus

erythematosus (SLE) at different pretest probabilities for a positive and negative cell-bound complement activation products (CB-CAPs) in multi-analyte assay with algorithm (MAAA) test result by antinuclear antibody (ANA) status (positive, titre $\geq 1: 40$; negative titre $<1: 40$ ). The post-test probability of a positive CB-CAP in MAAA test result was calculated using a sensitivity of $73 \%$ among ANA positive and $14 \%$ among ANA negative (specificity was set at $97.5 \%$ ). Specificity of $97.5 \%$ was used to calculate the post-test probability of a CB-CAP in MAAA negative test result. Results are presented by ANA positive or negative as determined using indirect immunofluorescence.

diagnosis of SLE when combined with routinely determined autoantibodies. ${ }^{18}$ In the present study, we have established the diagnostic performances of the test in patients with primary FM and SLE. Our results indicate that CB-CAPs in MAAA are highly specific for SLE. The CB-CAPs in MAAA was used unmodified in comparison with the original diagnostic methodology previously described and the present data therefore provide a prospective validation of our diagnostic test.

Because SLE is far less prevalent than FM, the vast majority of patients presenting with non-specific muscular, articular and constitutional symptoms will have FM and not SLE. ${ }^{32}$ Many clinicians rely on ANA testing to identify SLE but the ANA test has severe limitations including a significant number of false-positives. While these limitations are recognised by rheumatologists, many primary care physicians may be unaware of these drawbacks and inappropriate referral of ANA-positive patients can follow the initial clinical assessment. Because of its high specificity, the CB-CAPs in MAAA could potentially facilitate the appropriate referral of true patients with SLE to the rheumatologist.

A significant proportion of SLE subjects enrolled in this study were testing ANA negative and these data are consistent with previous observations in patients with established and treated disease. ${ }^{33}$ This relatively high incidence of ANA-negative subjects in this SLE cohort was also consistent with the lower incidence of positivity to anti-dsDNA (17\%) reduced low complement C3/C4 (23\%), and elevated CB-CAPs (43\%) in comparison with our previous population of patients with SLE. ${ }^{18}$ This probably explains why the overall sensitivity was $60 \%$ in this cohort in comparison with the $80 \%$ 
sensitivity we have observed previously. In fact, when the analysis was restricted to ANA-positive subjects only, the percentage positivity for the CB-CAPs in MAAA rose to $73 \%$.

While the accuracy of a diagnostic result is often reported in terms of sensitivity and specificity, there are several potential drawbacks with this approach as busy clinicians tend to overestimate the probability of a disease (and SLE in this case) when the assessment in positive. ${ }^{35}$ Another approach to report test results is to use positive predictive values and negative predictive values, but these estimates are highly dependent on the prevalence of the disease and can be also misleading. Here we have reported our results as positive and negative LRs, which are only dependent on sensitivity and specificity while independent of disease prevalence. ${ }^{35}$ Moreover, LR have the attractive feature of allowing the ordering physician to derive a post-test probability of disease as a function of the patient-specific pretest probability. The application of these Bayesian principles to the differential diagnosis of SLE versus FM revealed that a positive CB-CAPs in MAAA test resulted in a positive LR of greater than 24, thereby indicating that people with SLE are greater than 24 times more likely to test positive compared with FM. This high positive LR translated into a large impact as indicated by an almost $70 \%$ increase in the probability of SLE versus FM (post-test probability, when starting with a pretest probability of $20 \%)$. Conversely, the negative LR was 0.4 for the CB-CAPs in MAAA, thereby indicating that patients with FM were 2.5 times $(1 / 0.4)$ more likely to test negative than those with SLE. We also analysed the results by stratifying the LRs by ANA status. Our analysis revealed that among ANA-negative subjects the impact of a negative CB-CAPs in MAAA test results had limited impact on post-test probability (eg, negative $\mathrm{LR}=0.86$ ), as did the other measures (eg, low complement, table 3), while a negative test result lowered the post-test probability significantly among ANA-positive subjects. Conversely, a positive CB-CAP test results had strong impact on post-test probability irrespective of the ANA status. A potential limitation of the study is the enrolment of patients with long disease duration and it will be important to establish the performance of the test in the setting of pre-lupus. Also, the majority of subjects enrolled were Caucasian and the generalisability of the findings to African American populations will need to be evaluated. Our test has been available to rheumatologists since 2012 (in the USA only) and nearly 70000 CB-CAPs have been determined in our clinical laboratory. We believe that this practical tool with enhanced performances compared with traditional complement measure $(\mathrm{C} 3 / \mathrm{C} 4)$ can help establish a diagnosis for SLE. Moreover it is a practical measure of complement activation as blood specimen can be shipped overnight from the physician office to the laboratory. In summary, our data indicate that the CB-CAPs in MAAA test has utility in differentiating SLE from FM, and the value of the test is particularly significant among subjects that are ANA positive. The measurement of CB-CAPs in MAAA could facilitate the appropriate referral of symptomatic patients with a positive ANA to the rheumatologist, and thus help initiate appropriate course of treatment.

Acknowledgements The authors thank all subjects for participating in the study and Claudia Ibarra for the management of the Clinical laboratory. They also thank Duncan Pooling, Tyler O'Malley, Joanne Ligayon and the clinical coordinators at each of the sites (Jennifer Nelson and Ryan Cotter) for technical assistance and management of the clinical protocol. They also acknowledge Dr Arthur Weinstein for critical review of manuscript and constructive comments.

Contributors All authors participated in the design of the study, collection of data, analysis and final approval of the manuscript.

Funding Exagen Diagnostics.

Competing interests TD, DB and JC are employees of Exagen. DJW and SS have received research grants.

Patient consent Obtained.

Ethics approval Central IRB.

Provenance and peer review Not commissioned; externally peer reviewed.

Data sharing statement No additional data are available.

Open Access This is an Open Access article distributed in accordance with the Creative Commons Attribution Non Commercial (CC BY-NC 4.0) license, which permits others to distribute, remix, adapt, build upon this work noncommercially, and license their derivative works on different terms, provided the original work is properly cited and the use is non-commercial. See: http:// creativecommons.org/licenses/by-nc/4.0/

\section{REFERENCES}

1. Rahman A, Isenberg DA. Systemic lupus erythematosus. $N$ Engl J Med 2008;358:929-39.

2. Oglesby A, Korves C, Laliberté F, et al. Impact of early versus late systemic lupus erythematosus diagnosis on clinical and economic outcomes. Appl Health Econ Health Policy 2014;12:179-90.

3. Alarcón GS, McGwin G Jr, Roseman JM, et al. Systemic lupus erythematosus in three ethnic groups. XIX. Natural history of the accrual of the American College of Rheumatology criteria prior to the occurrence of criteria diagnosis. Arthritis Rheum 2004;51:609-15.

4. Helmick CG, Felson DT, Lawrence RC, et al. Estimates of the prevalence of arthritis and other rheumatic conditions in the United States. Part I. Arthritis Rheum 2008;58:15-25.

5. Somers EC, Marder W, Cagnoli P, et al. Population-based incidence and prevalence of systemic lupus erythematosus: the Michigan Lupus Epidemiology and Surveillance program. Arthritis Rheumatol 2014;66:369-78.

6. Vincent A, Lahr BD, Wolfe F, et al. Prevalence of fibromyalgia: a population-based study in Olmsted County, Minnesota, utilizing the Rochester Epidemiology Project. Arthritis Care Res (Hoboken) 2013;65:786-92.

7. Narain S, Richards HB, Satoh M, et al. Diagnostic accuracy for lupus and other systemic autoimmune diseases in the community setting. Arch Intern Med 2004;164:2435-41.

8. Bennett RM. Clinical manifestations and diagnosis of fibromyalgia. Rheum Dis Clin North Am 2009;35:215-32.

9. Crofford LJ. Pain management in fibromyalgia. Curr Opin Rheumatol 2008;20:246-50.

10. Tan EM, Cohen AS, Fries JF, et al. The 1982 revised criteria for the classification of systemic lupus erythematosus. Arthritis Rheum 1982;25:1271-7.

11. Satoh M, Chan EK, Ho LA, et al. Prevalence and sociodemographic correlates of antinuclear antibodies in the United States. Arthritis Rheum 2012;64:2319-27.

12. Dinerman H, Goldenberg DL, Felson DT. A prospective evaluation of 118 patients with the fibromyalgia syndrome: prevalence of Raynaud's phenomenon, sicca symptoms, ANA, low complement, and Ig deposition at the dermal-epidermal junction. $J$ Rheumatol 1986;13:368-73. 
13. Calvo-Alén J, Bastian HM, Straaton KV, et al. Identification of patient subsets among those presumptively diagnosed with, referred, and/or followed up for systemic lupus erythematosus at a large tertiary care center. Arthritis Rheum 1995;38:1475-84.

14. Liu CC, Kao AH, Hawkins DM, et al. Lymphocyte-bound complement activation products as biomarkers for diagnosis of systemic lupus erythematosus. Clin Trans/ Sci 2009;2:300-8.

15. Kalunian KC, Chatham WW, Massarotti EM, et al. Measurements of cell-bound complement activation products enhance diagnostic performance in systemic lupus erythematosus. Arthritis Rheum 2012;64:4040-7.

16. Yang $\mathrm{DH}$, Chang DM, Lai JH, et al. Usefulness of erythrocyte-bound $\mathrm{C} 4 \mathrm{~d}$ as a biomarker to predict disease activity in patients with systemic lupus erythematosus. Rheumatology (Oxford) 2009;48:1083-7.

17. Liu CC, Ahearn JM, Manzi S. Complement as a source of biomarkers in systemic lupus erythematosus: past, present, and future. Curr Rheumatol Rep 2004;6:85-8.

18. Putterman C, Furie R, Ramsey-Goldman R, et al. Cell-bound complement activation products in systemic lupus erythematosus: comparison with anti-double-stranded DNA and standard complement measurements. Lupus Sci Med 2014;1:e000056.

19. Petri M, Orbai AM, Alarcón GS, et al. Derivation and validation of the Systemic Lupus International Collaborating Clinics classification criteria for systemic lupus erythematosus. Arthritis Rheum 2012;64:2677-86.

20. Wolfe F, Smythe HA, Yunus MB, et al. The American College of Rheumatology 1990 Criteria for the Classification of Fibromyalgia. Report of the Multicenter Criteria Committee. Arthritis Rheum 1990;33:160-72.

21. Jolly M, Garris CP, Mikolaitis RA, et al. Development and validation of the Lupus Impact Tracker: a patient-completed tool for clinical practice to assess and monitor the impact of systemic lupus erythematosus. Arthritis Care Res (Hoboken) 2014;66:1542-50.

22. Bennett RM, Friend R, Jones KD, et al. The Revised Fibromyalgia Impact Questionnaire (FIQR): validation and psychometric properties. Arthritis Res Ther 2009;11:R120.
23. Petri M, Kim MY, Kalunian KC, et al. Combined oral contraceptives in women with systemic lupus erythematosus. $N$ Engl J Med 2005;353:2550-8.

24. Copple SS, Jaskowski TD, Giles R, et al. Interpretation of ANA indirect immunofluorescence test outside the darkroom using NOVA view compared to manual microscopy. J Immunol Res 2014;2014:149316.

25. Bossuyt X. Clinical performance characteristics of a laboratory test. A practical approach in the autoimmune laboratory. Autoimmun Rev 2009;8:543-8.

26. McGee S. Simplifying likelihood ratios. J Gen Intern Med 2002;17:647-50.

27. Grimes DA, Schulz KF. Refining clinical diagnosis with likelihood ratios. Lancet 2005;365:1500-5.

28. Hanley JA, Lippman-Hand A. If nothing goes wrong, is everything all right? Interpreting zero numerators. JAMA 1983;249:1743-5.

29. Eypasch E, Lefering R, Kum CK, et al. Probability of adverse events that have not yet occurred: a statistical reminder. $B M J$ 1995;311:619-20.

30. Brown LD, Cai TT, DasGupta A. Interval Estimation for a Binomial Proportion. Stat Sci 2001;16:101-33.

31. Agresti A, Coull BA. Approximate Is Better than "Exact" for Interval Estimation of Binomial Proportions. The American Statistician 1998;52:119-26.

32. Blumenthal DE. Tired, aching, ANA-positive: does your patient have lupus or fibromyalgia? Cleve Clin J Med 2002;69:143-2.

33. Wallace DJ, Stohl W, Furie RA, et al. A phase II, randomized, double-blind, placebo-controlled, dose-ranging study of belimumab in patients with active systemic lupus erythematosus. Arthritis Rheum 2009;61:1168-78.

34. Giles I, Isenberg D. Antinuclear antibodies: An overview. In: Wallace DJ, Hahn BH, eds. Dubois' Lupus Erythematosus. 7th edn. Lippincott Williams \& Wilkins, 2007:432-41.

35. Vermeersch $P$, Bossuyt $X$. Comparative analysis of different approaches to report diagnostic accuracy. Arch Intern Med 2010;170:734-5. 